Original Research Paper

\title{
The Effect of a Small Group Life Story Intervention with Rural Community Dwelling Older Adults
}

\author{
Lillian Felicia Jones and Mary A. Nies \\ School of Nursing, Division of Health Sciences, Idaho State University, United States
}

Corresponding Author:

Lillian Felicia Jones

School of Nursing, Division of

Health Sciences, Idaho State

University, United States

Email: ljones3610@charter.net

\begin{abstract}
To determine if a small group life story nursing intervention can be beneficial to rural community dwelling older adults by decreasing depression scores and increasing perceived social support scores. This study employed a quasi-experimental one group pretest/posttest design. This study was conducted in thirteen rural senior facilities with a community population of less than 20,000 in two western states in the United States. 137 English-speaking volunteers over age 60 years from 13 senior centers were screened for dementia and depression to determine eligibility for the study. Eligibility was established by a negative dementia screen and a score of four or greater on the Geriatric Depression Scale short version. Of the 137 volunteers, 40 were eligible and asked to participate in intervention groups, three centers had the most participants willing to participate in intervention groups, these groups were composed of four volunteers each. This study utilized the Geriatric Depression Scale short version and the Multidimensional Scale of Perceived Social Support for pre-and post-testing. The intervention consisted of four hourly group sessions involving storytelling about life events. No significant difference was noted between pretest and posttest scores based on the intervention. Participant evaluation of the small group activity indicated that increased social interaction was the greatest benefit derived from the intervention. The intervention did not significantly decrease depression scores or increase perceived social support scores; however, small group life story interventions may have utility in increasing social interaction and increasing social connections among rural older adults. It is recommended that future studies use a pre-existing support group for the small group intervention or members of a rural assisted living facility where depression rates can be high.
\end{abstract}

Keywords: Depression, Community, Rural, Older Adults, Life Story

\section{Introduction}

The process of telling one's story, or the creation of a life story book, has been used as a nursing intervention in the United Kingdom with people suffering from mental distress (Moya and Arnold, 2012); with dementia patients in Wales (Subramaniam et al., 2014); and in Norway to promote identity and dignity in long term care dementia patients, (Haggestad and Slettbo, 2015). These studies all used one-on-one interventions, were conducted in urban settings and conducted in private settings, long term care facilities, or psychiatric institutions. Novel mental health interventions are needed to serve rural dwellers who may have limited access to adequate health and mental healthcare. Small group interventions may have more utility in rural areas by gathering older adults together and decreasing the time and distance a nurse must travel to provide interventions. Using the small group setting for a storytelling intervention, specifically about life events and memories, may help to promote the older adult's personal identity, self-worth and increase social connection with peers, thereby decreasing the risk for depression. The purpose of this paper is to report the results of a research study that used a small group life story intervention with community dwelling older adults in the rural western United States.

\section{Background}

In the US, the over 60 population is growing and is predicted to reach 83.7 million by the year 2050 (USCB, 2010). Consequently, most people can expect to live 
long past the standard age for retirement. Along with increased longevity, older persons can expect to live longer with one or more chronic illness (Dexter et al., 2010). Chronic illness predisposes the older adult to depression (Taylor, 2014). The medications used treat these illnesses can contribute to or increase depressive symptoms as well (Taylor, 2014). Rural life presents additional risks for depression due to lack of resources for assessment and treatment, access and transportation issues, environmental factors and cultural beliefs, all of which can act as barriers to obtaining adequate care (Smalley et al., 2012; Winters, 2013).

Rural life challenges the desire of many rural dwelling older adults to stay in their homes and age comfortably in place. Besides decreased access to health care services, rural life can predispose older adults to social isolation (Nicholson, 2012). Social isolation is a notable health risk to all older adults and is associated with increased mortality and morbidity from physical and psychological illnesses (Nicholson, 2012). Mental health and substance abuse studies have indicated that by decreasing social isolation and increasing social connectedness, the recovery and entry of an individual into treatment can be enhanced (Mericle, 2014; Moya and Arnold, 2012). Rural health studies in Canada (Bascu et al., 2012) and Australia (Kelly, 2011), have noted that older adults living in rural areas who reported having the most social support and social connection also reported a greater sense of health and wellbeing. Therefore, providing a variety of interventions that are designed to increase social interaction and social support in rural older adults can help to promote mental well-being.

Many older adults believe that depression is a normal part of growing old (NIMH, 2015). Because it is a time of multiple losses, of friends, spouses, family members, employment and sometimes a person's home and daily routine, grieving is expected and normal to some extent. However, continuing sadness that affects a person's functioning or ability to perform daily activities for living, and/or further isolates the person, is not normal (NIMH, 2015). According to the NIMH (2015), depression is characterized by feelings of sadness, emptiness, hopelessness and anxiety, and can occur with Parkinson's disease, dementia, Alzheimer's Disease and many chronic illnesses such as cancer, diabetes and heart disease and the medications used to treat these diseases (NIMH, 2015). Elderly patients who are taking multiple medications are at increased risk for harmful complications when pharmaceutical treatments for depression are attempted and therefore, psychotherapy and non-pharmacological therapies are recommended as first line treatments (Taylor, 2014).
According to rural nursing theory, the rural dweller's concept of health lies in his/her ability to work and be functionally capable mentally, physically and emotionally (Winters, 2013). This concept of health is part of a culture that holds the values of steadfastness and self-reliance as desirable and therefore, rural folk may be suspicious of outsiders and help is often not accepted (Winters, 2013). Furthermore, the decreased privacy of small communities and the stigma associated with mental illness can often prevent an individual from admitting to having problems or asking for help (Kitchen et al., 2013). These factors of rural living, the culture, environment, access to services, the availability of assessment and treatment by a mental health provider, transportation and isolation, can affect the development, progression and outcome of a mental disorder in the older person.

In a longitudinal study using a life story intervention by Chan et al. (2013), older Chinese urban community dwellers with mild to severe depression reported decreased scores on the Geriatric Depression Scale short version (GDSsv; Yesavage, 1989) after each of the five sessions conducted over an eight-week period. Researchers attributed part of this reported decrease to the increased social interaction the intervention provided. The authors also noted that very few research studies had been done in the last ten years that supported life story as an intervention to decrease depression in older adults (Chan et al., 2013). In fact, only three studies were found, and all samples consisted of dementia patients not living in community settings (Chan et al., 2013).

\section{Theoretical Framework}

This research study employed concepts from Erikson's (1980) psychosocial development theory and Peplau's (1997) interpersonal theory. Erikson (1980) defines old age as a period of examination wherein a positive examination of a life well-lived can result in wisdom, or a negative examination from a life viewed as wasted, can result in despair. This stage in the theory helps explain the older adult's need for reminiscing and storytelling. Peplau's (1997) theory defines the nurse's role in the interaction with the older adult client. The nurse can take on several roles in this interaction: of counselor, by listening and providing encouragement; or as an advocate or resource for those who need to find help for mental distress; or as a teacher, instructing clients who have questions about their health, medications, or treatments. The nurse can utilize small group interventions as an aid to help promote wellbeing as well as increase social interactions among older adults.

\section{Research Questions}

This study was undertaken to determine if a small group life story nursing intervention could benefit rural 
community dwelling older adults who are at risk for a developing or worsening depression by increasing perceived social support. The study hypothesized that after participating in a four-session life story small group: 1) Geriatric Depression Scale short version scores (GDSsv; Yesavage, 1988) would decrease; and that, 2) Multidimensional Scale of Perceived Social Support (MSPSS; Ziamet et al., 1990) scores would increase in participants who took part in the intervention. The null hypothesis would be true if no significant change occurred in participant scores due to the intervention.

\section{Method}

\section{Design}

The study employed a quasi-experimental one group pretest/posttest design. Original plans for a control group had to be cancelled due to participant attrition; and therefore, only intervention group data was available for the pretest/posttest statistical analysis.

\section{Sample and Sampling Procedures}

After obtaining Institutional Review Board and individual senior center director approvals, a convenience sample of 137 volunteers were recruited through on-site advertising at thirteen rural community facilities that serve older adults. The senior facilities were in areas designated as rural by the United States Rural Urban Continuum Codes (RUCC) and consisted of towns with populations of less than 20,000 in two western states in the US. Recruitment occurred over a six-month period. Participants were adults 60 years of age and older and spoke English. After obtaining informed consent from participants, the nurse assistant established eligibility for the study by screening the individuals for cognitive function with the Mini-Cog Assessment (Borsen, 2000) and the Geriatric Depression Scale short version (GDSsv, Yesavage, 1988). Those with positive dementia screens and scores of less than four were excluded from the study. Of the 137 participants, 40 were determined to be eligible for the study with scores four or greater on the GDSsv and these individuals were asked to participate in the intervention. The three intervention groups consisted of a convenience sample of participants consenting to the intervention and were composed of four participants and two group leaders. Group leaders for the intervention were the nurse assistant and the researcher. The Multidimensional Scale of Perceived Social Support (MSPSS, Zimet et al., 1990), was also completed during pre-screening, but did not affect a volunteer's eligibility.

Participant recruitment was conducted at the investigator's portable booth near the entrance to the lunch room at the senior centers. The booth consisted of several tables and chairs to allow for inquiries and volunteer sign-ups. All study participants were assigned numbers for easy identification after obtaining consent. The nurse assistant obtained consent and collected demographic data on the participants which consisted of age, gender, marital status and race or ethnicity. All volunteers were informed by the nurse assistant that this information would be used solely for the purposes of describing the sample. All volunteers were asked to provide phone contact information for reminder calls about intervention group sessions dates and times.

\section{Instrumentation}

The Mini-Cog Assessment screening for dementia takes approximately three minutes and combines a threeitem recall with a clock drawing test (Borsen, 2000). The GDSsv consists of 15 items with yes/no answers and takes five to ten minutes (Yesavage, 1988). A score of zero to five is considered within normal limits, while scores greater than five indicate probable depression (Yesavage, 1988). This study used the score of four or greater to determine eligibility for participation in the groups; a similar determination was used by Chan et al. (2013). The GDSsv has been found to have a $92 \%$ sensitivity and $89 \%$ specificity when evaluated against diagnostic criteria, with the reliability and validity validated by research and clinical practice (Greenberg, 2012). The GDSsv was also found to have a high correlation $(r=0.84, \quad p<0.001)$ in differentiating depression from no depression in older adults (Greenberg, 2012). The MSPSS consists of a 12-item questionnaire on a Likert Scale ranging from 1-very strongly disagree to 7-very strongly agree. The items in the test are divided into three factor groups relating to the source of the social support: Family, friends and significant others and has a reported alpha of 0.88 for internal consistency (Zimet et al., 1990). The possible scores for this test range from 12 to 84, with 84 indicating maximum perceived social support.

\section{Data Collection}

After the initial assessment to determine eligibility and to obtain consent to participate in the intervention, group storytelling interventions commenced the following week. The number of sessions, intervention time and time allowed to complete the intervention sessions were modeled on the study by Chan et al. (2013), which as mentioned previously, consisted of five sessions completed over an eight-week period. Therefore, five visits to the senior centers were scheduled, one for the initial assessment (pretest) date and four hour-long intervention visits. Each intervention group was allowed a two-month period for completion and to allow for flexible scheduling around group member and facility availability. Although recruitment was ongoing over the six-month study, when an intervention session was scheduled for the week, recruitment days at other centers were not scheduled because of the long distances between centers. 
Prior to agreeing to participate in the intervention, prospective participants were informed by the researcher that the group would take an hour of their time for four weeks and that the group activity would consist of storytelling, specifically stories from their lives. These stories would progress from childhood to present day. As the goal of the intervention was to provide a positive experience for those involved in the group activity, some topics were taboo (i.e. no politics, religion or confidences that mandated nurse reporting).

The first intervention session consisted of a face to face instruction with the group members describing the intervention and the conditions of participation. The conditions of participation included notifying the group leader of any conflict with attending the sessions, courtesy for the person speaking, confidentiality about group sessions and stories heard during the sessions, and the goal of making all sessions a positive experience for group members. The group members were made aware of precautions in place should a participant react negatively to any story content which included: the participant being escorted to a private room by the group leader's assistant, the session discontinued for the day and the participant excluded from the study. Information about local mental health resources were made available to all participants. A follow-up call to the participant from the group leader was planned should an adverse event occur.

At the first session, after informing about the group activity and conditions of participation, the group storytelling intervention commenced. The group leader initiated the intervention by giving an example of an early childhood memory along with displaying a photo of the time-period. Group members were then encouraged to take turns telling the group members a little about themselves, such as where they were born, in what time-period, city or country and one experience they had as a child.

The progression of the group sessions was designed to move along a life time line from childhood to adulthood. Because of time constraints and to allow for full participation from all members, participants were asked to recount only one to two per session. The group leader acted as a mediator and time keeper to allow for equal sharing.

After the first session, participants were encouraged to bring their own photos to talk about for the second session. The second session continued where the first session left off and included recollections from their adolescence periods, while the third session included recollections from the participants' 20's, 30's and 40'. Although the sessions were structured to recall events from a single time period per session, if a participant recalled a story from an earlier time period, they were allowed to tell the story. As the main objective was to promote a positive experience for all group members, storytelling sessions were lightly structured. Employing a less-structured strategy allowed for greater participation without making members feel obligated to stay on a specific topic, and keep with the goal of each participant sharing at least one story with the group that day.

The fourth and final session included storytelling about recent events in participants' lives, a postassessment (posttest) and a written participant evaluation of the intervention. The post-assessment or posttest was completed at the end of the session and consisted of the GDSsv and the MSPSS administered by the nurse assistant. The written participant evaluation consisted of three questions: what type of benefit did you receive from this activity; what did you like or dislike about the activity; and, what would you do differently? These evaluations were anonymous, collected by a third party not involved with the study and were used to determine small group life story intervention use for future groups.

\section{Analysis}

Data analysis was done using the SPSS v22 (IBM, 2013) program. Descriptive statistics were used to describe the sample. Pretest-posttest scores were analyzed by t-tests and the effect sizes measured. All tests were run with a significance level of $p<0.05$.

\section{Results}

One hundred thirty-seven older adults volunteered and were screened for the study. These volunteers consisted of $86 \%$ Caucasian, $80 \%$ female, $20 \%$ male, $69 \%$ single and $75 \%$ between the ages of 60 and 80 . Based on GDSsv scores, $71 \%$ of this initial sample were not eligible for the study, while of the remaining $29 \%$, only three centers had enough eligible participants agreeing to the intervention. Three intervention groups resulted. These were composed of four participants and were predominantly female and Caucasian and between the ages of 60 and 90. All three groups had one Native American participant, one group had a married male participant and all groups had two married female participants.

Upon visual inspection, pre-and post-intervention participant GDSsv and MSPSS scores looked promising, indicating there may have been a decrease in depression scores due to the intervention. However, in comparison tests of before and after group means conducted by paired sample t-tests to determine if a significant difference exists, results were not so promising: On the GDSsv measure, the mean difference $(M=1.44, S D=$ 2.98) from pretest means to posttest means was not statistically significant, $t(2)=0.84, S E=1.72, p=0.49$, Cohen's $d=0.48$; while, on the MSPSS measure, the difference $(M=3.03, S D=11.39)$ from pretest means to posttest means was also not statistically significant, $t(2)$ $=0.46, S E=6.58, p=0.69$, Cohen's $d=0.27$ (Table 1). These results did not support the research hypothesis and therefore, the null hypothesis was applicable: depression scores did not decrease, and perceived social support scores did not increase due to the intervention alone. 
Table 1: Means and standard deviations for intervention groups $(\mathrm{N}=3)$

\begin{tabular}{lllll}
\hline Group & Pretest 1 & Pretest 2 & Posttest 1 & Posttest 2 \\
& $\mathrm{M}(\mathrm{SD})$ & $\mathrm{M}(\mathrm{SD})$ & $\mathrm{M}(\mathrm{SD})$ & $38.00(22.70)$ \\
\hline 1 & $5.00(2.00)$ & $48.00(24.26)$ & $7.00(3.00)$ & $79.25(9.50)$ \\
2 & $5.75(1.50)$ & $71.25(15.39)$ & $2.50(1.91)$ & $79.33(8.08)$ \\
3 & $5.75(1.71)$ & $68.25(13.94)$ & $2.67(2.52)$ & 196.58 \\
Total & 16.5 & 187.5 & 12.17 & $65.53(23.84)$ \\
Grand & $5.50(0.43)$ & $62.50(12.65)$ & $4.06(2.55)$ &
\end{tabular}

Note: Pretest, Posttest $1=$ Geriatric Depression Scale short version (Yesavage, 1988); Pretest, Posttest $2=$ Multidimensional Scale of Perceived Social Support (Zimet et al., 1990)

\section{Summary of Participant Evaluations}

At the last session, intervention participants were asked to complete an evaluation of the intervention to determine the utility of the intervention for future research. The first two questions produced similar responses to the benefit and what a person liked about the study. Typical responses for these questions indicated increased social interaction by increased socializing, making new friends and increasing motivation to meet new people. According to the evaluations, for some participants the intervention helped to form tighter bonds with others in their senior community, and an increased interest in community volunteering and participation in community events. The third question produced concerns about increasing participation and interest in the group.

\section{Discussion}

This study implemented a novel nursing intervention with rural community dwelling older adults. It is the first study that utilized a small group in a rural area for a life story intervention and it is the only life story group intervention done with rural community dwelling older adults. Past studies have utilized one-on-one life story interventions in private settings, long term care, or mental health facilities (Russel and Timmons, 2009; Thompson, 2011; Subramaniam et al., 2012; Haggestad and Slettebo, 2014; Moya and Arnold, 2012).

While the results of this study do not indicate a significant decrease in depression scores and perceived social support scores due to the intervention, participant evaluations indicated that the intervention increased participant social interaction. This finding supports the findings of Bascu et al. (2013; Kelly, 2011), which links increasing social interaction to the promotion of wellbeing in the older adult. It also supports the supposition by Chan et al. (2013), in that the decreasing depression scores seen over the course of their study may be due to the increased social interaction and attention given to the participant by the researcher. The study also contributes to knowledge supporting the psychiatric theory of depression proposed by Feely and Long (2009). The authors theorized that a lack of social connection can contribute to worsening symptoms of depression, and although this study did not find significant decreases in depression scores, it also did not find worsening depression scores.
In comparing results to that of the Chan et al. (2013) study, in which posttests were done after each session, this study's outcome may have been different had there been more than one posttest. It is possible that posttesting after each session in this study may have shown significant decreases in depression since the goal of each group session was to promote positive feelings.

The intervention itself also did not cause a significant increase in perceived social support scores and this could have been for one simple reason: perceived social support may be entirely linked to life events and if there is no change in life events, there may never be a change in perceived social support. Also, one's perception may be entirely dependent on their definition of social support. An example of this is the low score from one participant that indicated that all her family and friends were deceased and that she did not perceive to have a support system before or after the group despite the intervention.

\section{Limitations}

\section{Design}

Attrition and a lack of random assignment affected the experimental design. The plan for a control group had to be cancelled due to high participant attrition and instead, a one group pre-and post-intervention design was implemented. One group pre/post intervention designs have minimal internal validity and no external validity. Therefore, the study cannot be generalized to other older adult populations because it consisted of a convenience sample of participants that were willing to participate and may have excluded those persons suffering from depression but refused to participate. In addition, the willing participants in the intervention groups were predominantly Caucasian and female. These factors should be taken into consideration when determining the significance of the results. A dominating presence of one sex, race, age group, or single persons may affect the outcome of any group experience.

\section{Rural Barriers}

The long distances between recruiting sites and the research base ranged from thirty minutes to five hours one way. This resulted in a limited number of centers 
that could be visited for recruiting purposes weekly and a limited number of intervention groups that could be conducted daily. Some recruitment issues that were experienced can be attributed to rural culture; previous studies note that rural culture values self-reliance and tends to be suspicious of help or intrusion by outsiders (Smalley et al., 2012; Winters, 2013). While most of the older adults attending the senior centers were polite and inquisitive about the research booth and what the research entailed, many were suspicious and reluctant to volunteer, provide any identifying information (including demographic information), or sign the informed consent. Suspicion continued to linger despite detailed explanations about the study, the purpose of the study, and even after participating in an intervention group. One participant phoned the researcher several times after the intervention sessions to verify the study's legitimacy, about the need for demographic information and how older adults would benefit from the study.

While suspicion may be the reason for some refusals, other aspects of rural culture can account for participant ineligibility. The values of self-reliance and steadfastness, resistance to accepting help from outsiders and the stigma of mental illness may prevent individuals from admitting to a problem, or perpetuate the belief that depression is normal, or that the problem is something that can be dealt with amongst family. Also, because the depression questionnaire relies on a certain amount of honesty on the part of the volunteer, it is totally possible that many were not truthful and denied depressive symptoms when in fact they were experiencing symptoms of depression.

\section{Participant Bias}

In situations where a bond is formed between participants and the researcher, biases can develop. In this case, there was increased familiarity with the researcher because the researcher was also the group leader for the small group intervention; and, although this was anticipated from the beginning and determined to be beneficial in promoting social interaction, bias could have influenced the participants' responses to the post-intervention evaluation. The conscious or subconscious desire to help the researcher may explain greater positive versus negative comments made on the evaluative surveys.

Because of possible participant bias, group attendance can offer verification regarding the liking or disliking of an activity: If a group member is frequently absent, one could assume the participant disliked the activity. In this case, all participants voiced their commitment to the group and if they could not make a date, sessions were rearranged so everyone could be present.

\section{Small Groups}

Previous studies have used the one-on-one approach for life story-telling and this may be more advantageous for some individuals because small groups can present as a threatening situation. This may be one reason a participant may refuse to take part in the intervention; while other reasons can include protection of privacy, and shyness or anxiety when involved in group interactions. One intervention group member wrote on the evaluation that it was difficult to tell her stories because she feared rejection from group members initially, but as she became more comfortable with the group, she could talk more about her life experiences.

\section{Conclusion}

This study implemented a novel nursing intervention with older adults living in rural areas. Although quantitative results indicated that there were no significant changes in the depression scores or the perceived social support scores post-intervention, survey evaluations of the intervention support the small group storytelling intervention for increasing social interaction and social connection in rural community dwelling older adults. For nursing, finding novel interventions that increase social interaction and work to decrease social isolation, a major risk factor for depression, is important to the promotion of health and wellbeing among rural community dwelling older adults.

\section{Recommendations for Future Research}

Because rural barriers affect recruitment and research on rural older adults, future studies may need to focus on members of pre-existing support groups or rural assisted living facilities where depression rates can be quite high (Allen, 2015).

\section{Acknowledgement}

The authors would like to acknowledge the nurse assistant, Lucie Palumbo RN, for her help in collecting data.

\section{Author's Contributions}

Lillian Felicia Jones: Conception and design, acquisition of data, analysis and interpretation of data, drafting of original and revisions of article.

Mary A. Nies: Contribution to design, critical examination of intellectual content, critical review of original article and all revisions.

\section{Ethics}

This article is original and contains unpublished material, no ethical issues involved. 


\section{References}

Allen, J., 2015. Depression in assisted living. Geriatric Nursing, 36: 78-80. DOI: 10.1016/J.GERINURSE.2014.12.005

Bascu, J., B. Jeffrey, S. Ebony, S. Johnson and N. Novak et al., 2013. Healthy aging in place: Perceptions of rural older adults. Educ. Gerontol., 40: 327-337. DOI: 10.1080/03601277.2013.802191

Borsen, S., 2000. The mini-cog: A cognitive "vital signs" measure for dementia screening in multi-lingual elderly. Int. J. Geriatric Psychiatry, 15: 1021-1021.

Chan, M.F., S.E. Ng, A. Tien, R.C.M. Ho and J. Thayala, 2013. A randomized controlled study to explore the effect of life story review on depression in older Chinese in Singapore. Health Soc. Care Community, 21: 545-553. DOI: 10.1111/hsc.12043

Dexter, P.R., D.K. Miller, D.O Clark, M. Weiner and L.E. Harris et al., 2010. Preparing for an aging population and improving chronic disease management. Proceedings of the AMIA Annual Symposium, (AMI' 2010), pp: 162-166.

Erikson, E.H., 1980. Elements of a psychoanalytic theory of psychosocial development. The course of life: Psychoanalytic contributions toward understanding personality development.

Feely, M. and A. Long, 2009. Depression: A psychiatric nursing theory of connectivity. J. Psychiatric Mental Health Nursing, 16: 725-737. DOI: $10.1111 / \mathrm{j} .1365-2850.2009 .01452 . \mathrm{x}$

Greenberg, S.A., 2012. The Geriatric Depression Scale (GDS). Hartford Institute Geriatric Nursing.

Haggestad, A.K. and A. Slettebo, 2015. How individuals with dementia in nursing homes maintain their dignity through life story telling: A case study. J. Clin. Nursing, 24: 2323-2330. DOI: 10.1111/jocn.12837

Kelly, B.J., 2011. Determinants of mental health and well-being within rural and remote communities. Soc. Psychiatry Psychiatric Epidemiol., 46: 1331-1342.

Kitchen, A.K., C.L. McKibben, T.L. Wykes, A.A. Lee and C.P. Carrico et al., 2013. Depression treatment among rural older adults: Preferences and factors influencing future service use. Clin. Gerontol., 36: 241-259. DOI: 10.1080/07317115.2013.767872
Mericle, A., 2014. The role of social networks in recovery from alcohol and drug abuse. Am. J. Drug Alcohol Abuse, 40: 179-180. DOI: $10.3109 / 00952990.2013 .875553$

Moya, H. and P. Arnold, 2012. A life story toolkit to support recovery from mental Distress. Mental Health Practice, 16: 14-18.

NIMH, 2015. Older adults and depression.

Nicholson, N.R., 2012. A review of social isolation: An important but under assessed condition in older adults. J. Primary Prevention, 33: 137-152.

DOI: $10.1007 /$ s 10935-012-0271-2

Peplau, H.E., 1997. Peplau's theory of interpersonal relations. Nursing Sci. Quarterly, 10: 162-167. DOI: $10.1177 / 089431849701000407$

Russel, C. and C. Timmons, 2009. Life story work and nursing home residents with dementia. Nursing Older People, 21: 28-32.

Subramaniam, P., B. Woods and C. Whitaker, 2012. Life review and life story books for people with mild to moderate dementia. Aging Mental Health, 18: 363-375. DOI: 10.1080/13607863.2013.837144

Taylor, W.D., 2014. Depression in the elderly. New England J. Med., 371: 1228-1237. DOI: $10.1056 /$ NEJMcp 1402180

Thompson, R., 2011. Using life story work to enhance care. Nursing Older People, 23: 16-21.

Yesavage, G., 1988. The geriatric depression scale (short version). Pharmacol. Bull., 24: 709-711.

Smalley, K., W. Bryant and J. Rainer, 2012. Rural Mental Health: Issues, Policies and Best Practices. 1st Edn., Springer, New York.

Winters, C., 2013. Rural Nursing: Concepts, Theory and Practice. 4th Edn., Springer, New York.

Zimet, G.D., S.S. Powell, G.K. Farley, S. Workman and K.A. Berkoff, 1990. Psychometric characteristics of the multidimensional scale of perceived social support. J. Personality Assessment, 55: 610-617. DOI: 10.1080/00223891.1990.9674095 